CPT-94/P.3106

Mannheimer Manuskripte 181

November 1994

$[181$.

\title{
A generalized Lichnerowicz formula, the Wodzicki Residue and Gravity
}

\author{
Thomas Ackermann ${ }^{1}$ and Jürgen Tolksdorf ${ }^{2}$ * \\ ${ }^{1}$ Fakultät für Mathematik \& Informatik, Universität Mannheim \\ D-68159 Mannheim, F.R.G \\ 2 Centre de Physique Théorique, CNRS Luminy, Case 907 \\ F-13288 Marseille Cedex 9, France
}

\begin{abstract}
A bstract. We prove a generalized version of the well-known Lichnerowicz formula for the square of the most general Dirac operator $\widetilde{D}$ on an even-dimensional spin manifold associated to a metric connection $\widetilde{\nabla}$. We use this formula to compute the subleading term $\Phi_{1}\left(x, x, \widetilde{D}^{2}\right)$ of the heat-kernel expansion of $\widetilde{D}^{2}$. The trace of this term plays a key-rôle in the definition of a (euclidian) gravity action in the context of non-commutative geometry. We show that this gravity action can be interpreted as defining a modified euclidian Einstein-Cartan theory.
\end{abstract}

Keywords: non-commutative geometry, Lichnerowicz formula, Dirac operator, heat-kernelexpansion, Wodzicki residue, gravity

1 e-mail: ackerm@euler.math.uni-mannheim:de

2 e-mail: tolkdorf@cptsu4.univ-mrs.fr

* Supported by the European Community, contract 
Zugangsinummer: 853194 Signatur:

UNIVERSITÄT MANNHEIM Bibl d. Fok. I. Nathematik u. Informatik 


\section{Introduction}

When attempting to quantize the electron in 1928, Dirac introduced a first-order operator the square of which is the so-called wave-operator (d'Alembertian operator). Lateron, in the hands of mathematicians generalizations of this operator, called 'Dirac operators' - evolved into an important tool of modern mathematics, occuring for example in index theory, gauge theory, geometric quantisation etc.

More recently, Dirac operators have assumed a significant place in Connes' noncommutative geometry $[\mathrm{C}]$ as the main ingredient in the definition of a $\mathrm{K}$-cycle. Here they encode the geometric structure of the underlying non-commutative 'quantumspaces'. Thus disguised, Dirac operators re-enter modern physics, since non-commutative geometry can be used, e.g. to derive the action of the standard model of elementary particles, as shown in [CL] and [K1]. Initially, it remains unclear wether it was possible to also derive the Einstein-Hilbert action of gravity using this approach. And again, it was a Dirac operator which proved to be the key to answer this question. According to Connes [C], the 'usual' Dirac operator $D$ on the spinor bundle $S$ associated to the Levi-Civita connection on a four-dimensional spin manifold $M$ is linked to the euclidian Einstein-Hilbert gravity action via the Wodzicki residue of the inverse of $D^{2}$. This was shown in detail in [K2]. A further question that naturally arises is the the dependence of this result from the chosen Dirac operator $D$. In other words, does $\operatorname{Res}\left(\widetilde{D}^{-2}\right)$ change if $\widetilde{D}$ is a Dirac operator on $S$ different from the 'usual' one?

In this paper we answer this question affirmatively. Moreover, in section 3, we compute the lagrangian of an appropriatly defined gravity action

$$
I_{G R}(\widetilde{D}):=-\frac{2}{2^{n}(2 n-1)} \operatorname{Res}\left(\tilde{D}^{-2 n+2}\right)
$$

for the most general Dirac operator $\widetilde{D}$ associated to a metric connection $\widetilde{\nabla}$ on a compact spin manifold $M$ with $\operatorname{dim} M=2 n \geq 4$. We proceed as follows; According to the main theorem of $[\mathrm{KW}]$, there is a relation between the Wodzicki residue $\operatorname{Res}\left(\hat{\triangle}^{-n+1}\right)$ of a generalized laplacian $\hat{\triangle}$ on a hermitian bundle $E$ over $M$ and 
$\Phi_{1}(x, x, \hat{\triangle})$, which denotes the the subleading term of its the heat-kernel expansion. It is well-know that, given any generalized Laplacian $\hat{\triangle}$ on $E$, there exists a connection $\hat{\nabla}^{E}$ on $E$ and a section $F$ of the endomorphism bundle End $(E)$, such that $\hat{\triangle}$ decomposes as

$$
\hat{\triangle}=\Delta^{\hat{\nabla}^{E}}+F
$$

However, this has a slight flaw. The decomposition (1.2) neither provides any method to construct the connection $\hat{\nabla}^{E}$ nor the endomorphism $F$ explicitly. Nevertheless, it is exactly this endomorphism $F \in \Gamma(\operatorname{End} E)$ which fully determines the subleading term $\Phi_{1}(x, x, \hat{\triangle})$ of the heat-kernel-expansion of $\hat{\triangle}$ (cf. [BGV]). Thus the the problem of computing $\operatorname{Res}\left(\widehat{\triangle}^{-n+1}\right)$ is transformed into the problem of computing $F$.

For an arbitrary general laplacian, this might prove to be difficult. However, in the case where $E=S$ and the general laplacian $\hat{\triangle}$ is the square $\widetilde{D}^{2}$ of a Dirac operator associated to an arbitrary metric connection $\widetilde{\nabla}$ on $T M$, a constructive version of (1.2) can be proved. This will be shown in section 2 . Because of its close relationship to the well-known Lichnerowicz formula (cf. [L]) we call our decomposition formula a 'generalized Lichnerowicz formula'. We understand it as beein intrinsic to the the Dirac operators studied in this paper.

As already mentioned, we will compute the lagrangian of (1.1) in section 3, using our generalized Lichnerowicz formula as the main technical tool. From a physical point of view, this lagrangian can be interpreted as defining a modified (euclidian) Einstein-Cartan theory.

\section{A generalized Lichnerowicz formula}

Let $M$ be a spin manifold with $\operatorname{dim} M=2 n$ and let us denote its Riemannian metric by $g$.. The Levi-Civita connection $\nabla: \Gamma(T M) \rightarrow \Gamma\left(T^{*} M \otimes T M\right)$ on $M$ induces a a connection $\nabla^{S}: \Gamma(S) \rightarrow \Gamma\left(T^{*} M \otimes S\right)$ on the spinor bundle $S$ which is compatible with the hermitian metric $<\cdot, \cdot>_{S}$ on $S$. By adding an additional torsion term 
$t \in \Omega^{1}(M, \operatorname{End} T M)$ we obtain a new covariant derivative

$$
\widetilde{\nabla}:=\nabla+t
$$

on the tangent bundle $T M$. Since $t$ is really a one-form on $M$ with values in the bundle of skew endomorphism $S k(T M)$ (cf. [GHV]), $\widetilde{\nabla}$ is in fact compatible with the Riemannian metric $g$ and therefore also induces a connection $\widetilde{\nabla}^{S}=\nabla^{S}+T$. on the spinor bundle. Here $T \in \Omega^{1}(M, \operatorname{End} S)$ denotes the 'lifted' torsion term $t \in \Omega^{1}(M, \operatorname{End} T M)$. However, in general this induced connection $\widetilde{\nabla}^{S}$ is neither compatible with the hermitian metric $<\cdot, \cdot>_{S}$ nor compatible with the Clifford action on $S$. With respect to a local orthonormal frame $\left\{e_{a}\right\}_{1 \leq a \leq 2 n}$ of $\left.T M\right|_{U \subset M}$ we have

$$
\begin{array}{cc}
\nabla_{c} e_{b}=\omega_{b c}^{a} e_{a} & t:=t_{b c}^{a} e_{a} \otimes e^{b} \otimes e^{c} \\
\nabla^{S} s_{l}=\frac{1}{4} \gamma^{a} \gamma^{b} s_{l} \otimes \omega_{a b c} e^{c} & \tilde{\nabla}^{S} s_{l}=\frac{1}{4} \gamma_{l}^{a} \gamma^{b} s_{l} \otimes\left(\omega_{a b c}+t_{a b c}\right) e^{c}
\end{array}
$$

where $\omega_{b c}^{a}$ denotes the components of the Levi-Civita connection, $\left\{e^{a}\right\}_{1 \leq a \leq 2 n}$ the corresponding dual frame of $\left\{e_{a}\right\}_{1 \leq a \leq 2 n}$ and $\left\{s_{l}\right\}_{1 \leq l \leq \operatorname{dim} S}$ a local frame of $\left.S\right|_{U}$. Nnote that we use the following conventions

$$
\left\{\gamma^{a}, \gamma^{b}\right\}=-2 \eta^{a b}, \quad\left[\gamma^{a}, \gamma^{b}\right]=2 \gamma^{a b}
$$

for the representation $\gamma: C_{\mathbb{C}}\left(T^{*} M\right) \rightarrow$ End $S$ of the complexified Clifford algebra of $T^{*} M$ on the spinor bundle.

We now define by $\widetilde{D}:=\gamma^{\mu} \widetilde{\nabla}_{\mu}^{S}$ a first order operator $\widetilde{D}: \Gamma(S) \rightarrow \Gamma(S)$ associated to the metric connection $\widetilde{\nabla}$. Since $\widetilde{D}$ satisfies the relationship $[\widetilde{D}, f]=\gamma^{\mu} \frac{\partial f}{\partial x^{\mu}}$ for all $f \in C^{\infty}(M)$ this operator $\widetilde{D}$ is a Dirac operator, i.e. its square $\widetilde{D}^{2}$ is a generalized laplacian (cf. [BGV]). Note that $\widetilde{D}$ is also the most general Dirac operator on the spinor bundle $S$ corresponding to a metric connection $\widetilde{\nabla}$ on $T M$.

According to the well-known Ricci lemma (cf. [GHV]) there is a one-to-one correspondence between metric connections on $T M$ and the elements of $\Omega^{1}(M, S k(T M))$. Consequently, the set of all such Dirac operators acting on sections of the spinor bundle $S$ over $M$ is parametrized by $t \in \Omega^{1}(M, S k(T M))$. 
For the square of the Dirac operator $\widetilde{D}$ we get the following standard decomposition

$$
\widetilde{D}^{2}=-g^{\mu \nu} \tilde{\nabla}_{\mu}^{S} \widetilde{\nabla}_{\nu}^{S}+\gamma^{\mu}\left[\widetilde{\nabla}_{\mu}^{S}, \gamma^{\nu}\right] \widetilde{\nabla}_{\nu}^{S}+\frac{1}{2} \gamma^{\mu} \gamma^{\nu}\left[\widetilde{\nabla}_{\mu}^{S}, \widetilde{\nabla}_{\nu}^{S}\right]
$$

If $t=0$, which means that $\widetilde{\nabla}$ is identical with the Levi-Civita connection $\nabla$, equation (2.2) is the first step to compute the well-known Lichnerowicz formula of $D^{2}$, cf. $[\mathrm{L}]$. Note that none of the first two terms of (2.2) is covariant in itself but only their sum. Using (2.1) we can, however, rearrange the decomposition (2.2) such that each term is manifest covariant. Moreover the derivations in the decomposition will then be arranged according to their degree:

LEMMA 2.1. Let $M$ be a spin mannifold, $\nabla$ the Levi-Civita connection on $T M$ and $\widetilde{\nabla}$ defined by $\widetilde{\nabla}:=\nabla+t$ with $t \in \Omega^{1}(M, S k(T M))$. Then the square $\widetilde{D}^{2}$ of the Dirac operator $\widetilde{D}$ on the spinor bundle $S$ associated to $\widetilde{\nabla}$ decomposes as

$$
\widetilde{D}^{2}=\triangle^{\nabla}-B^{\mu} \nabla_{\mu}^{S}+F^{\prime}
$$

where $B: \Gamma(T M \otimes \operatorname{End} S)$ and $F^{\prime} \in \Gamma(\operatorname{End} S)$ are defined by

$$
\begin{aligned}
B^{a} & :=2 T^{a}-\gamma^{c}\left[T_{c}, \gamma^{a}\right] \\
F^{\prime} & :=\frac{1}{4} R^{\nabla} \cdot \mathbb{I}_{\text {End } S}+\gamma^{a} \gamma^{b}\left(\nabla_{a} T_{b}\right)+\gamma^{a} T_{a} \gamma^{b} T_{b}
\end{aligned}
$$

Furthermore $R^{\nabla}$ denotes the scalar curvature and $\triangle^{\nabla}:=\eta^{a b}\left(\nabla_{a}^{S} \nabla_{b}^{S}-\nabla_{\nabla_{a} e_{b}}^{S}\right)$ the horizontal laplacian on the spinor bundle corresponding to the Levi-Civita connection $\nabla$ with respect to a local orthonormal frame $\left\{e_{a}\right\}_{1 \leq a \leq 2 n}$.

Proof: By inserting $\tilde{\nabla}_{\mu}^{S}=\nabla_{\mu}^{S}+T_{\mu}$ in (2.2) and using the compatibility of the connection $\nabla^{S}$ with the Clifford action, so that $\left[\nabla_{\mu}^{S}, \gamma^{\sigma}\right]=-\gamma^{\nu} \Gamma_{\nu \mu}^{\sigma}$ we get

$$
\begin{aligned}
\widetilde{D}^{2}= & \Delta^{\nabla}+\frac{1}{2} \gamma^{\mu \nu}\left[\nabla_{\mu}^{S}, \nabla_{\nu}^{S}\right]-g^{\mu \nu}\left(\left[\nabla_{\mu}^{S}, T_{\nu}\right]-\Gamma_{\nu \mu}^{\sigma} T_{\sigma}\right) \\
& -g^{\mu \nu} T_{\mu} T_{\nu}-g^{\mu \nu}\left(2 T_{\mu} \nabla_{\nu}^{S}\right)+\gamma^{\mu}\left[T_{\mu}, \gamma^{\nu}\right] \nabla_{\nu}^{S}+\frac{1}{2} \gamma^{\mu \nu}\left[T_{\mu}, T_{\nu}\right] \\
& +\gamma^{\mu \nu}\left[\nabla_{\mu}^{S}, T_{\nu}\right]+\gamma^{\mu}\left[T_{\mu}, \gamma^{\nu}\right] T_{\nu}
\end{aligned}
$$


With the help of the Clifford relation $\gamma^{\mu} \gamma^{\nu}+\gamma^{\nu} \gamma^{\mu}=-2 g^{\mu \nu}$ and the first Bianchi identity $R_{j k l i}+R_{k l i j}+R_{l i j k}=0$ one can identify the second term in (2.6) with the 'usual' Lichnerowicz term:

$$
\frac{1}{2} \gamma^{\mu \nu}\left[\nabla_{\mu}^{S}, \nabla_{\nu}^{S}\right]=\frac{1}{4} R R^{\nabla} \cdot \mathbb{1}_{S}
$$

If we write $\gamma^{\mu}\left[T_{\mu}, \gamma^{\nu}\right] \nabla_{\nu}=g^{\mu \nu} \gamma^{\sigma}\left[T_{\sigma}, \gamma_{\mu}\right] \nabla_{\nu}$, we see that $B^{\nu}$ is given by the sum of the fifth together with the sixth term on the right-hand-side. Furthermore we have the identities

$$
\begin{array}{r}
-g^{\mu \nu}\left(\left[\nabla_{\mu}^{S}, T_{\nu}\right]-\Gamma_{\mu \nu}^{\sigma} T_{\sigma}\right)=-g^{\mu \nu}\left(\left(\nabla_{\mu}^{\text {End } S} T_{\nu}\right)-\Gamma_{\mu \nu}^{\sigma} T_{\sigma}\right)=-g^{\mu \nu}\left({ }^{\prime} \nabla_{\mu} T_{\nu}\right) \\
\gamma^{\mu \nu}\left[\nabla_{\mu}^{S}, T_{\nu}\right]=\gamma^{\mu \nu}\left(\left(\nabla_{\mu}^{\text {End } S} T_{\nu}\right)-\Gamma_{\mu \nu}^{\sigma} T_{\sigma}\right)=\gamma^{\mu \nu}\left({ }^{\prime} \nabla_{\mu} T_{\nu}\right) .
\end{array}
$$

Here $\left.{ }^{\prime} \nabla: \Gamma\left(\operatorname{End} S \otimes T^{*} M\right) \rightarrow \Gamma^{(} T^{*} M \otimes \operatorname{End} S \otimes T^{*} M\right)$ denotes the induced connection ${ }^{\prime} \nabla:=\nabla^{\text {End } S} \otimes \mathbb{I}_{T^{*} M}+\mathbb{I}_{\text {End } S} \otimes \nabla$ on the tensor bundle End $S \otimes T^{*} M$. Because $\nabla$ respects the Clifford relation this means that

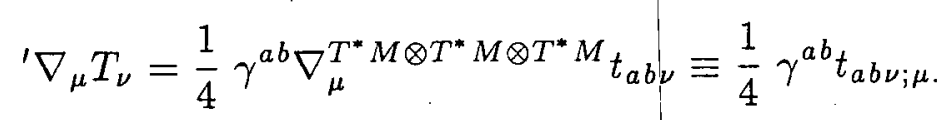

Due to the fact that $\gamma^{\mu \nu}-g^{\mu \nu}=\gamma^{\mu} \gamma^{\nu}$ we obtain our result.

It is well-know (see [BGV]) that given any generalized Laplacian $\hat{\triangle}$ on a hermitian bundle $E$ over $M$, there exists a connection $\hat{\nabla}^{E}$ on $E$ and a section $F$ of the endomorphism bundle End $(E)$, such that $\hat{\triangle}$ decomposes as

$$
\hat{\triangle}=\Delta^{\hat{\nabla}^{E}}+F \text {. }
$$

As we have mentioned before, this statement does not offer any possibility of calculating the endomorphism $F$ explicitly. Since it can be shown (cf. [BGV]), however, that

$$
\Phi_{1}(x, x, \hat{\triangle})=\frac{1}{6} R^{\nabla} \cdot \mathbb{1}_{E}-F
$$

it is evident that $F$ plays a leading rôle in the computation of the subleading term $\Phi_{1}(x, x, \hat{\triangle})$ in the asymptotic expansion of the heat kernel of $\hat{\triangle}$. Moreover, by the main theorem the main theorem of $[\mathrm{KW}]$

$$
\operatorname{Res}\left(\hat{\triangle}^{-n+1}\right)=\frac{2 n-1}{2} \int_{M} * \operatorname{tr}\left(\Phi_{1}(x, x, \hat{\triangle})\right)
$$

(1) We denote by * the Hodge-star operator associated to the Riemannian metric $g$. 
this endomorphism $F$ also determines the Wodzicki residue of $\widehat{\triangle}^{-n+1}$ which defines gravity actions in the case of $\hat{\triangle}=\widetilde{D}^{2}$.

We shall now prove a theorem which enables us to compute $F$ explicitly in the case of $E=S$ and $\hat{\triangle}:=\widetilde{D}^{2}$.

THEOREM 2.2. Let the hypotheses be the same as in lemma 2.1 and let $\hat{\triangle}=\widetilde{D}^{2}$ be the square of the Dirac operator $\widetilde{D}$ associated to $\widetilde{\nabla}$. Then the covariant derivative $\hat{\nabla}^{S}$ and the endomorphism $F \in \Gamma($ End $S)$ in the decomposition (2.8) are defined as follows:

$$
\begin{aligned}
\hat{\nabla}^{S} & :=\nabla^{S}+\hat{T} \\
F & :=F^{\prime}+\aleph .
\end{aligned}
$$

With respect to a local orthonormal frame $\left\{e_{a}\right\}_{1 \leq a \leq n}$ of $T M, \hat{T} \in \Omega^{1}(M, \operatorname{End} S)$ and $\aleph \in \Gamma(\operatorname{End} S)$ are explicitly given by

$$
\begin{gathered}
\hat{T}_{a}=T_{a}-\frac{1}{2} \gamma^{b}\left[T_{b}, \gamma_{a}\right] \\
\aleph={ }^{\prime} \nabla_{a} \hat{T}^{a}+\hat{T}_{a} \hat{T}^{a}
\end{gathered}
$$

where $F^{\prime} \in \Gamma(\operatorname{End} S)$ is the endomorphism (2.5) of lemma 2.1 .

Proof: The main ingredient of this proof is the global decomposition formula (2.3) of $\widetilde{D}^{2}$ as given in lemma 2.1. Concerning the case of $\widehat{\triangle}=\widetilde{D}^{2}$, equation (2.3) is but an alternative version of (2.8). We can therefore prove the theorem by inserting (2.10), (2.11), (2.12) and (2.13) into equation (2.8) which then is identical with (2.3).

$\square$

Thus we obtain the following formula for the square of the Dirac Operator $\widetilde{D}$ :

$$
\begin{aligned}
\widetilde{D}^{2}=\triangle^{\nabla^{s}} & +\frac{1}{4} R \cdot \mathbb{I}_{S}+\gamma^{a b}\left(\nabla_{a} T_{b}\right)+\frac{1}{2} \gamma^{a b}\left[T_{a}, T_{b}\right] \\
& +\frac{1}{2}\left[\gamma^{a}\left[T_{a}, \gamma^{b}\right], T_{b}\right]-\frac{1}{2} \gamma^{b}\left[\left({ }^{\prime} \nabla_{a} T_{b}\right), \gamma^{a}\right] \\
& +\frac{1}{4} \eta_{a b} \gamma^{c}\left[T_{c}, \gamma^{a}\right] \gamma^{d}\left[T_{d}, \gamma^{b}\right] .
\end{aligned}
$$


In the case of $t=0$, i.e. $T=0$, this decomposition obviously reduces to the usual Lichnerowicz formula $D^{2}=\Delta^{\nabla}+\frac{1}{4} R^{\nabla} \cdot \mathbb{I}_{S}$. Consequently, we call (2.14) a 'generalized Lichnerowicz formula'.

Notice that one has to take into acount that in general it is impossible to find any $S k(T M)$-vallued one-form $\hat{t} \in \Omega^{1}(M, S k(T M))$ such that the endomorphism part $\hat{T}_{X} \in \operatorname{End} S$ of $\hat{T}$ corresponds to $\hat{t}_{X} \in S k(T M)$ for all $X \in \Gamma(T M)$. Hence, $\hat{\nabla}^{S}$ is generally not induced by any metric connection $\hat{\nabla}$ on $T M$ in general. However, if $t \in \Omega^{1}(M, S k(T M)$ is totally antisymmetric we obtain the following

LEMMA 2.3. Let $M$ be a spin mannifold, $\nabla$ the Levi-Civita connection on $T M$ and $\tilde{\nabla}$ defined by $\tilde{\nabla}:=\nabla+t$ where $t \in \Omega^{1}(M, S k(T M))$ is totally antisymmetric. Then $\hat{T}=3 T$ and consequently $\hat{\nabla}^{S}=\nabla^{S}+3 T$.

This can simply derived from the definiton (2.12) of $\hat{T}$.

\section{Euclidian Gravity}

According to Connes [C] there exists a link between the usual Dirac operator $D:=$ $\gamma^{\mu} \nabla_{\mu}^{S}$ on the spinor bundle $S$ of a four-dimensional spin manifold $M$ associated to the Levi-Civita connection and the euclidian Einstein-Hilbert gravity action via the Wodzicki residue $\operatorname{Res}\left(D^{-2}\right)$ of the inverse of $D^{2}$. This was explicitly verified in $[\mathrm{K}]$. Moreover, as already mentioned, the main theorem of $[\mathrm{KW}]$ states that the the Wodzicki residue $\operatorname{Res}\left(\hat{\triangle}^{-n+1}\right)$ of any generalized laplacian $\hat{\triangle}$ acting on sections of an hermitian vector bundle $E$ over an even-dimensional manifold $M$ with $\operatorname{dim} M=2 n \geq 4$ can be identified with

$$
\frac{2 n-1}{2} \int_{M} * \operatorname{tr}\left(\Phi_{1}\left(x, x, \hat{\triangle}^{\prime}\right)\right) .
$$

Again $\Phi_{1}(x, x, \hat{\triangle})$ denotes the subleading term of the asymtotic expansion of the heat-kernel of $\hat{\triangle}$. In this sense a gravity action can be defined by an arbitrary Dirac operator $\widetilde{D}:=\gamma^{\mu} \widetilde{\nabla}_{\mu}^{S}$ on $S$ associated to a metric connection $\widetilde{\nabla}$ on the base $M$, this 
means

$$
I_{\mathrm{GR}}(\widetilde{D}):=-\frac{1}{2^{n}} \int_{M} * \operatorname{tr}\left(\Phi_{1}\left(x, x, \widetilde{D}^{2}\right) .\right.
$$

Here $2^{n}=\operatorname{dim}_{\mathbb{C}} S$ is the complex dimension of the spinor bundle. By using our generalized Lichnerowicz formula (2.14), we can now easily compute $\operatorname{tr}\left(\Phi_{1}\left(x, x, \widetilde{D}^{2}\right)\right)$. All that remains to be done is to take traces of $\gamma$-matrices. Thus, we obtain the

LeMma 3.1. Let $M$ be a spin manifold with $\operatorname{dim} M=2 n$ even and $\widetilde{D}: \Gamma(S) \rightarrow$ $\Gamma(S)$ the Dirac operator on the spinor bundle $S$ associated to a metric connection $\widetilde{\nabla}:=\nabla+t$ as above. Then

$$
-\frac{1}{2^{n}} \operatorname{tr}\left(\Phi_{1}\left(x, x, \widetilde{D}^{2}\right)\right)=\frac{1}{12} R^{\nabla}+\frac{1}{2^{n}}\left(-t_{a b c} t^{a b c}+2 t_{a b c} t^{a c b}\right)
$$

with respect to a local orthonormal frame of $T M$.

Note that this result (3.2) holds independently of wether or not the corresponding Dirac operator $\widetilde{D}$ is self-adjoint with respect to the hermitian metric on the spinor bundle $S$. In the special case of the torsion tensor being totally anti-symmetric, (3.2) reduces to $-\frac{1}{2^{n}} \operatorname{tr}\left(\Phi_{1}\left(x, x, \widetilde{D}^{2}\right)\right)=\frac{1}{12} R^{\nabla}-\frac{3}{2^{n}} t_{[a b c]} t^{[a b c]}$ as already shown in [KW].

In order to find out wether (3.2) defines a pure (euclidian) Einstein-Cartan theory (2) we express the right-hand side of (3.2) by the scalar curvature $R^{\widetilde{\nabla}}$ of $\widetilde{\nabla}$. Using the well-known formula $\mathbf{R}^{\widetilde{\nabla}}=\mathbf{R}^{\nabla}+d^{\nabla} t+\frac{1}{2}[t \wedge t]$, where $\mathbf{R}^{\widetilde{\nabla}} \in \Omega^{2}(M$, End $T M)$ denotes the curvature of $\widetilde{\nabla}$ and $d^{\nabla}$ is the exterior covariant derivative corresponding to the Levi-Civita connection $\nabla$, we can rewrite (3.2) as follows

$$
\begin{aligned}
-\frac{1}{2^{n}} \operatorname{tr}\left(\Phi_{1}\left(x, x, \widetilde{D}^{2}\right)\right)= & \frac{1}{12} R^{\widetilde{\nabla}}+\frac{1}{12} t_{a b c t} t^{b c a}-\frac{1}{2^{n}} t_{a b c} t^{a b c}+\frac{1}{2^{n-1}} t_{a b c} t^{a c b} \\
& +\frac{1}{12} t_{a b}{ }^{b} t^{a}{ }_{c}^{c}-\frac{1}{12} \nabla_{\mu} t_{a}^{\mu}{ }_{a}^{a} .
\end{aligned}
$$

Without additional mater fields, our result (3.2) obviously reduces to the usual Einstein theory of gravity. Hence we obtain a result similar to that in [KW]. We also conclude from (3.3) that it is not possible to obtain a 'pure' Einstein-Cartan theory

(2) By an Einstein-Cartan theory we understand a gravity theory based on the Einstein-Hilbert action. 
from the square of an arbitrary Dirac operator $\sim$ associated to a metric connection on $M$ by using the Wodzicki residue.

\section{Conclusion}

In this paper we proved a generalized version of the well-known Lichnerowicz formula for the most general Dirac operator $\widetilde{D}$ on the spinor bundle of an evendimensional spin manifold $M$ associated to a metric connection $\widetilde{\nabla}$ on $T M$. Applying this formula, the subleading term $\Phi_{1}\left(x, x, \widetilde{D}^{2}\right)$ of the heat-kernel expansion of $\widetilde{D}^{2}$ is easy to compute. According to [KW], the trace of this term plays a key-rôle in the definition of a (euclidian) gravity action $I_{G R}(\widetilde{D})$ in the context of the noncommutative differential geometry à introduced by Connes [C]. This gravity action can be interpreted as defining a modified Einstein-Cartan theory.

Finally, we would like to add that it is also possible to derive a combined EinsteinHilbert/Yang-Mills lagrangian from an appropriatly defined Dirac operator by using similar techniques. Moreover, this Dirac operator can be considered as a deformation of the well-known Dirac-Yukawa operator. This will be shown in a forthcoming paper $[\mathrm{AT}]$.

Acknowledgements. We are indebted to T. Schücker for his stimulating support. We would also like to thank Susanne for carefully reading the manuscript. One of us, T.A. would like to thank E. Binz for his gentle support.

\section{Refererences}

[AT] T.Ackermann, J.Tolksdorf, Super-connections and a combined Einstein-Yang-Mills la-

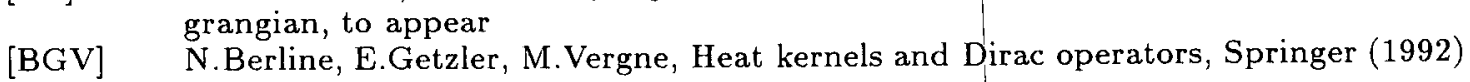


[C] A.Connes, Non-commutative geometry and physics, IHES preprint (1993)

[CL] A.Connes, J.Lott,Particle models and non-commutaive geometry,Nucl. Phys. B Proc. Supp. 18B (1990) 29-47

[GHV] W.Greub, S.Halperin, R.Vanstone, Connections, curvature and cohomology, vol. 1, Academic press (1976)

[KW] W.Kalau, M.Walze, Gravity, non-Commutative Geometry and the Wodzicki residue, to appear in Journ. of Geometry and Physics

[L] A.Lichnerowicz, Spineurs harmonique, C. R. Acad. Sci. Paris Sér. A 257 (1963)

[K1] D.Kastler, A detailed account of Alain Connes' version of the standard model in noncommutative differential geometry I, II and III,to appear in Rev. Math. Phys.

[K2] D.Kastler, The Dirac operator and gravitation, to appear in Com. Math. Phys. 